\title{
Plea Bargaining \\ in the \\ United States
}

by

Herbert S. Miller

William F. McDonald

James A. Cramer

September 1978

National Institute of Law Enforcement and Criminal Justice Law Enforcement Assistance Administration

U.S. Department of Justice 